\title{
Nanomechanical identification of liquid reagents in a microfluidic channel
}

\author{
Khan, Faheem; Kim, Seonghwan; Lee, Dongkyu; Schmid, Silvan; Boisen, Anja; Thundat, Thomas
}

Published in:

Lab on a Chip

Link to article, DOI:

10.1039/c3lc51273h

Publication date:

2014

Document Version

Publisher's PDF, also known as Version of record

Link back to DTU Orbit

Citation (APA):

Khan, F., Kim, S., Lee, D., Schmid, S., Boisen, A., \& Thundat, T. (2014). Nanomechanical identification of liquid reagents in a microfluidic channel. Lab on a Chip, 14(7), 1302-1307. https://doi.org/10.1039/c3lc51273h

\section{General rights}

Copyright and moral rights for the publications made accessible in the public portal are retained by the authors and/or other copyright owners and it is a condition of accessing publications that users recognise and abide by the legal requirements associated with these rights.

- Users may download and print one copy of any publication from the public portal for the purpose of private study or research.

- You may not further distribute the material or use it for any profit-making activity or commercial gain

- You may freely distribute the URL identifying the publication in the public portal 


\title{
Nanomechanical identification of liquid reagents in a microfluidic channel $\dagger$
}

\author{
M. Faheem Khan, $\neq^{\text {ab }}$ Seonghwan Kim, $t^{\text {bc }}$ Dongkyu Lee, ${ }^{\text {b }}$ Silvan Schmid, ${ }^{a}$ \\ Anja Boisen ${ }^{\star a}$ and Thomas Thundat ${ }^{* b^{*}}$
}

Integration of promising technologies that can enhance sensitivity, selectivity, and throughput into micro total analysis systems ( $\mu$ TAS) are important in making them useful in precise screening of reaction byproducts in analytical chemistry, cellular biology and pharmaceutical industries. But unfortunately so far a method to precisely determine molecular signatures of reagents is missing in $\mu$ TAS. We have developed a technique whereby molecular signatures of $50 \mathrm{pL}$ of liquid reagents confined within a bimetallic microchannel cantilever can be obtained. This is achieved using wavelength dependent mechanical bending of the cantilever under infrared (IR) radiation. This technique also allows simultaneous physical characterization of the liquid reagent using variations in resonance frequency. It is useful in lab-on-a-chip devices and has a myriad of applications in drug screening, bioreactor monitoring, and petrochemical analysis.

Received 14th November 2013, Accepted 6th January 2014

DOI: 10.1039/c3lc51273h

www.rsc.org/loc structures by the groups of Stemme ${ }^{5}$ and Manalis, ${ }^{6}$ allows ultrasensitive mass measurements for biomolecules, nanoparticles, microbes, and cells in liquid, as well as liquid reagents using mechanical resonance. ${ }^{6-10}$ This breakthrough, which places the liquid inside a resonator instead of using the conventional method of placing the resonator in a liquid, overcomes the limitation of damping-induced low sensitivity in the mass-based detection. Although it adds extremely high sensitivity to mass density ${ }^{11-13}$ and viscosity detection, ${ }^{14,15}$ it does not provide chemical selectivity. Second, recent advances in nanomechanical calorimetric infrared (IR) spectroscopy of adsorbed materials using a bi-material cantilever offer high selectivity detection without relying on extrinsic labels or specific receptors. ${ }^{16}$ However, this technique has been limited to solid samples placed on the cantilever and could not be used for liquid analytes.

We combined the two processes described above to develop a spectroscopic technique which is capable of realtime molecular recognition of picoliter volumes of reagents confined within the suspended resonating cantilever. In a $\mu$ TAS, this technique presents an additional module for a real-time, highly selective molecular level analysis.

The precise identification of molecular signatures of small amounts of liquids is challenging because of sensors generally with low sensitivity, low selectivity or long analysis time. The current state of the art techniques for detection of molecules dissolved in liquid are surface plasmon resonance ${ }^{17}$ (SPR), silicon nanowires ${ }^{18}$ (SNW), suspended microchannel resonators ${ }^{19}$ (SMR), quartz crystal monitors ${ }^{20}$ (QCM) and liquid chromatography $^{21}$ (LC). Except for SMR, all of the above 
stated techniques need liquids in microliters to perform an analysis. Additionally the analysis time is also in several minutes. SMR is considered quite quick with an analysis time of one minute while consuming liquids in picoliters. But unfortunately it can only measure the physical properties of the reagents. Moreover, to measure molecular signatures of liquids, conventional Fourier transform (FT) spectroscopic techniques such as FTIR and FT-Raman are also in practice. Their sensitivity is too low for small volumes of reagents and it is challenging to develop a low cost, high throughput analysis system by employing such methods.

We have developed a bi-material microchannel cantilever (BMC) which needs $50 \mathrm{pL}$ volume of a liquid reagent to perform physical as well as chemical analysis. As compared to the previously mentioned techniques, the BMC has a potential to characterize low volumes of liquid reagents with comparable sensitivity. At a specific wavelength it has an analysis time of less than a minute. Moreover, BMC chips can be used in an array configuration to speed up analysis of multiple reagents.

The BMC can be potentially used in micro-bioreactors where researchers need to have high throughput analysis devices to find the right combination of bacteria and biomass which produces the most glucose and eventually ferments glucose to ethanol. ${ }^{22}$ Moreover, the BMC can be a potential candidate in analyzing cellular contents of microalgae where real-time monitoring of production of different chemicals from microalgae is required. ${ }^{23}$ Another strongly anticipated use of the BMC is the detection of circulating microvesicles (100 $\mathrm{nm}$ structures shed from tumor cells) which exist in low concentration in blood but have very distinct molecular signatures. $^{24}$ The BMC can also be used in pharmaceuticals where characterization of preformulations can be made cheaper by consuming smaller volumes of drugs. ${ }^{25}$

\section{Experimental}

In the presented method, a picoliter volume of fluid sample, contained in the microfluidic channel on top of a bi-material microcantilever, absorbs IR photons at a certain wavelength. Then it releases the energy to the phonon background of the bi-material cantilever through multiple steps of vibrational energy relaxation. These nonradiative decay processes result in a minute temperature change in the bi-material cantilever causing a measurable deflection (Fig. 1a). The nanomechanical IR spectrum represents the molecular vibration signature (Fig. 1b) while the resonance frequency change provides real time information regarding the mass density of the fluid sample (Fig. 1c). Since the volume of the microfluidic channel on top of the cantilever is fixed, the mass of the fluid sample can be directly determined with density-frequency calibration measurements. $^{13}$

A U-shaped microfluidic channel (16 $\mu \mathrm{m}$ wide, $1050 \mu \mathrm{m}$ long and $3 \mu \mathrm{m}$ high) is fabricated on top of a plain cantilever (44 $\mu \mathrm{m}$ wide, $500 \mu \mathrm{m}$ long and $500 \mathrm{~nm}$ thick). ${ }^{26}$ This cantilever structure is turned into a bi-material beam by depositing a $500 \mathrm{~nm}$ thick layer of aluminum on its bottom side using e-beam evaporation. This bi-material microchannel cantilever is supported on a $350 \mu \mathrm{m}$ thick silicon chip, which provides fluidic inlets and outlets for delivering sample fluids to the microchannel on the cantilever (Fig. 2a). Two openings at the bottom of the chip provide a fluidic interface between the chip and connecting Teflon tubes (Fig. 2b).

The successful characterization of a reagent also depends upon its evaporation rate. For liquids with high vapour pressure, it is important to properly seal the BMC before taking a measurement. This is achieved by packaging the BMC chip in a holder made of polyether ether ketone (PEEK). The holder provides connections to larger tubes for the delivery
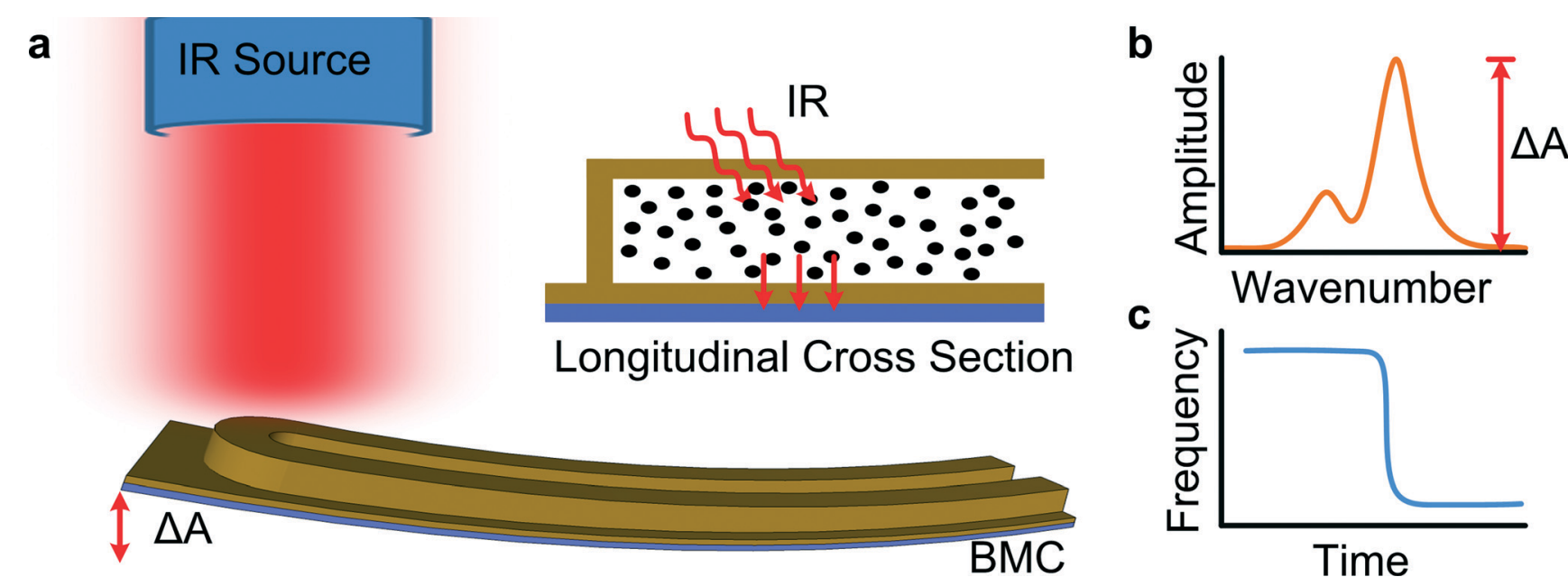

Fig. 1 (a) Using a tunable source, a $500 \mu \mathrm{m}$ long and $44 \mu \mathrm{m}$ wide BMC is irradiated with IR light (spot diameter: $4 \mathrm{~mm}$ ). The longitudinal cross section shows the microchannel of the BMC when filled with a sample. As the molecules of the sample absorb IR radiation at their characteristic resonance frequency, localized heat is generated as a result of a nonradiative decay process. As a result of different rates of thermal expansion of aluminum (in blue) and silicon nitride (in golden), the BMC deflects upwards. (b) A precise nanomechanical IR spectrum of a sample can be generated by plotting the amplitude of nanomechanical deflection of the BMC as a function of IR wavenumber. (c) Orthogonal measurement of mass density of a reagent is possible by monitoring the resonance frequency of the BMC. 


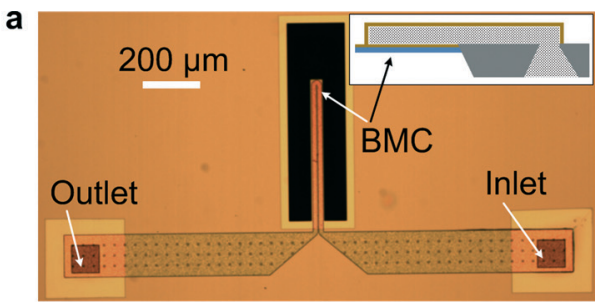

b

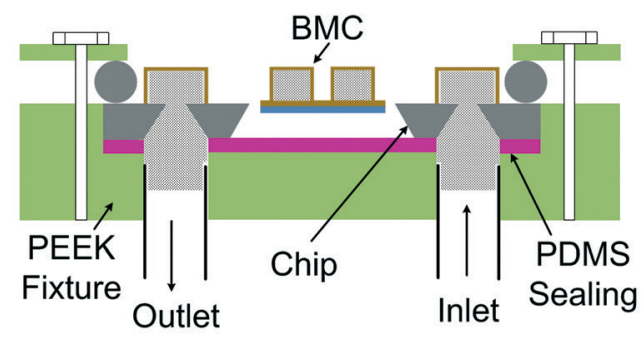

C

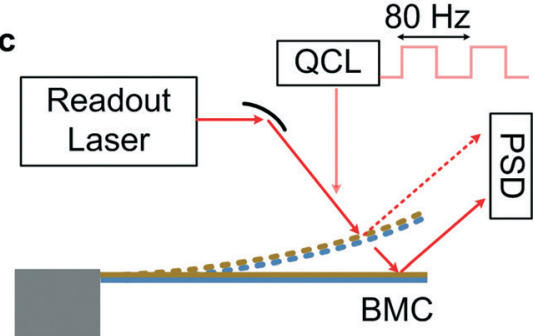

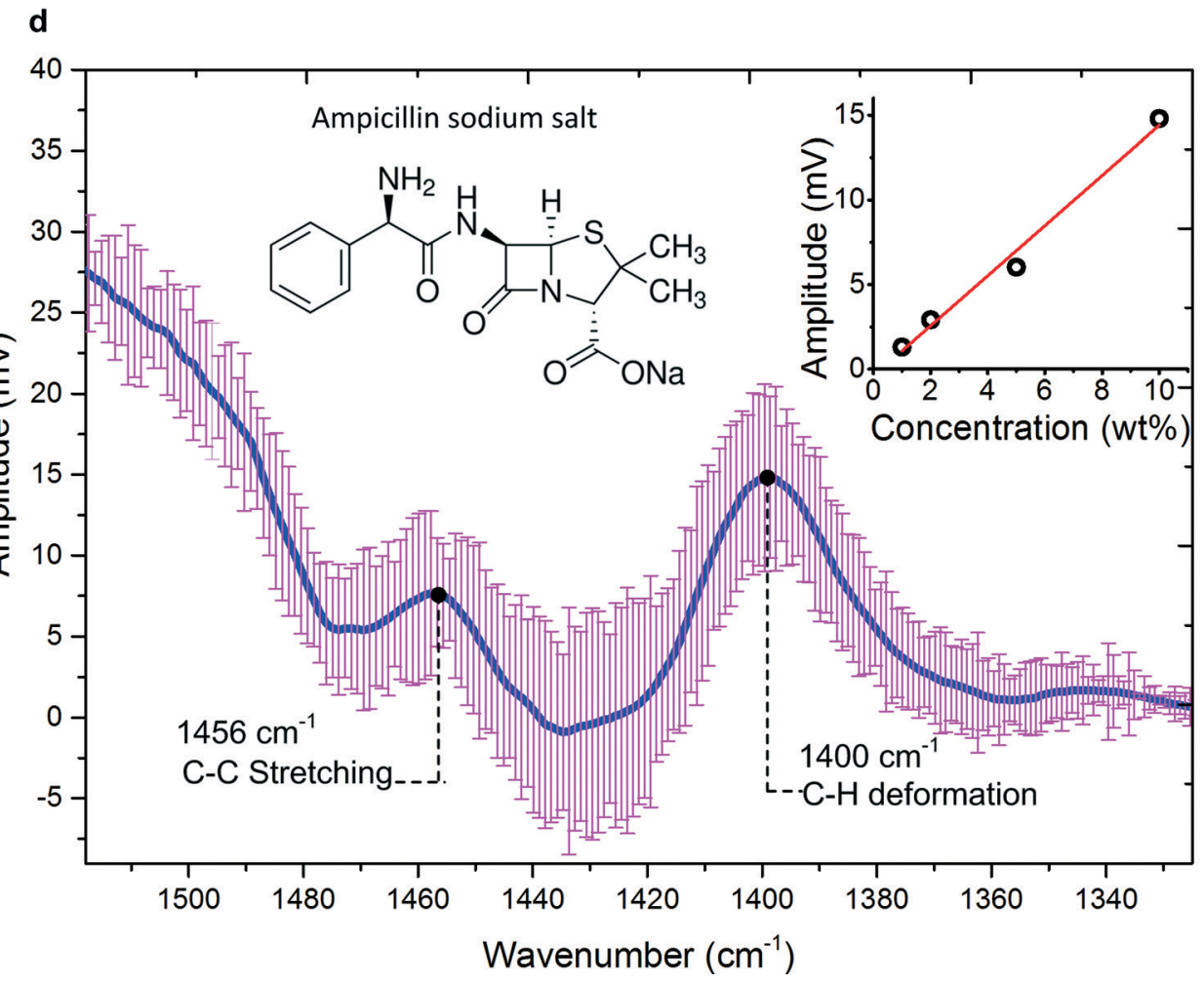

Fig. 2 (a) Top view of a chip containing the BMC, sample delivery channels and inlet/outlet. The inset provides a side view showing the microchannel (in gold), the metal layer (in blue) and the substrate (in grey). (b) The chip is packaged in a PEEK fixture through which the inlet of the chip is connected with a sample reservoir and the outlet is connected with a syringe pump. (c). Using a tunable quantum cascade laser, the BMC is irradiated with a series of different wavelengths of IR light. The deflection of the BMC is measured by reflecting a visible laser (635 nm) to a position sensitive detector (PSD). For simplicity, the microchannel is not shown on top of the cantilever. (d) The nanomechanical IR spectrum of 10 wt\% ampicillin sodium salt, an antibacterial drug, is obtained using the BMC. The inset on the right shows a linear relationship in nanomechanical deflection (at $1400 \mathrm{~cm}^{-1}$ ) as a function of the ampicillin sodium salt concentrations $(1,2.5,5$ and 10 wt\%). More data is presented in the ESI. $\dagger$

of fluid samples to the BMC. A sealed contact between the PEEK holder and the chip is achieved using a polydimethylsiloxane (PDMS) gasket. To load the fluid sample inside the BMC, a syringe pump is connected at the outlet tube in order to create a negative pressure which causes the fluid sample to be pulled from inlet to outlet, passing through the BMC. Since the microfluidic channels are optically transparent, the fluid sample entering the BMC can be visually observed using an optical microscope.

\section{IR radiation}

External-cavity quantum cascade lasers (QCLs) (Daylight Solutions Inc.) are used as the sources of IR light. The advantages of 
using QCLs over thermal IR sources are: pulsed operation (up to $200 \mathrm{kHz}$ ), high optical power (up to $500 \mathrm{~mW}$ peak power), room temperature operation, broad tunability, high spectral resolution (down to $0.1 \mathrm{~nm}$ ), and compact assembly. For our experiments, three QCLs are used; MIRCat ${ }^{\mathrm{TM}}$ (bandwidth: $6.3 \mu \mathrm{m}$ to $12.5 \mu \mathrm{m}$, i.e. $1587 \mathrm{~cm}^{-1}$ to $800 \mathrm{~cm}^{-1}$ ); ÜT-7 (bandwidth: $6.4 \mu \mathrm{m}$ to $7.4 \mu \mathrm{m}$, i.e. $1540 \mathrm{~cm}^{-1}$ to $1345 \mathrm{~cm}^{-1}$ ); and ÜT-8 (bandwidth: $7.1 \mu \mathrm{m}$ to $8.7 \mu \mathrm{m}$, i.e. $1408 \mathrm{~cm}^{-1}$ to $1145 \mathrm{~cm}^{-1}$ ). During IR scanning, the resonance frequency and deflection of the BMC are measured using an optical lever technique whereby a probing red laser (with a spot size of approximately $50 \mu \mathrm{m}$ ) is reflected onto a position sensitive detector (PSD) as shown in Fig. 2c. The four quadrant PSD converts the optomechanical signal to a recordable electronic signal. The ÜT-8 QCL is pulsed at $200 \mathrm{kHz}$ while the ÜT-7 and MIRCat ${ }^{\mathrm{TM}}$ are pulsed at $100 \mathrm{kHz}$. The pulse frequency is further modulated using an optimized burst frequency of $80 \mathrm{~Hz}$ which is generated by a lock-in-amplifier. The cantilever is therefore exposed to an IR pulse every 6.25 milliseconds. This time period is enough to provide thermal relaxation to the BMC. To find the amplitude of a signal at $80 \mathrm{~Hz}$, the $y$-axis signal of the PSD is fed into a lock-in-amplifier (SR850) (Stanford Research Systems). In order to continuously measure the resonance frequency of the cantilever, a spectrum analyzer (SR760) (Stanford Research Systems) is used to measure the fast Fourier transform (FFT) of the $y$-axis signal of the PSD. An oscilloscope is used to monitor and keep the laser spot in the centre of the PSD's sensitive area. The data from the lock-in-amplifier and the spectrum analyzer are stored in a computer using a data acquisition card and Labview. The signal is then plotted with respect to the wavenumber of IR light, which generates a nanomechanical IR spectrum of the analyte inside the BMC.

\section{Results and discussion}

To demonstrate the capability of the BMC-based calorimetric IR microspectroscopy, we obtained the nanomechanical IR spectra of aqueous solutions of ampicillin sodium salt $\left(\mathrm{C}_{16} \mathrm{H}_{18} \mathrm{~N}_{3} \mathrm{NaO}_{4} \mathrm{~S}\right)$, an antimicrobial drug agent. The spectra corresponding to the four concentrations of the drug $(1,2.5$, 5 , and $10 \mathrm{wt} \%$ ) in deionized water are collected. In order to acquire the nanomechanical IR spectra, each sample is loaded separately into the BMC by applying a negative pressure at the inlet of the chip. As the drug exists as a solid phase, it is initially dissolved in deionized water. This makes the sample loading quite convenient. Using the QCL MIRCat ${ }^{\mathrm{TM}}$, the $\mathrm{BMC}$ is then irradiated with IR light from $1518 \mathrm{~cm}^{-1}$ to $1325 \mathrm{~cm}^{-1}$. Initially as a background, the IR spectrum of the BMC (filled with deionized water) is measured which is followed by the measurement on the BMC filled with a mixture of deionized water and ampicillin powder. Later differential nanomechanical IR spectra are achieved by subtracting the background spectra from the ones with an analyte. All of the measurements are performed at atmospheric pressure and room temperature.
Several distinct peaks and shoulders appear in the IR spectra. Two strong absorption peaks at $1456 \mathrm{~cm}^{-1}$ and at $1400 \mathrm{~cm}^{-1}$ (Fig. 2d) are attributed to aromatic C-C stretching and $\mathrm{C}-\mathrm{H}$ deformation, respectively. The inset on the right in Fig. 2d shows the amplitudes of the nanomechanical IR absorption peaks at $1400 \mathrm{~cm}^{-1}$ as a function of the ampicillin sodium salt concentration. The straight line indicates a linear fit of peak amplitudes. Such a fitting can be used for calibration to measure the concentration of molecules dissolved in liquids. The spectra of the ampicillin solutions with concentrations of 1, 2.5 and $5 \mathrm{wt} \%$ are presented in the ESI. $\dagger$ The limit of detection for ampicillin sodium salt at $1400 \mathrm{~cm}^{-1}$ is estimated to be $0.6 \mathrm{wt} \%$ with a signal-to-noise ratio of 3 . However, to acquire nanomechanical calorimetric peaks at $1456 \mathrm{~cm}^{-1}$ below $10 \mathrm{wt} \%$, an improved design of the BMC is required. For comparison, the FTIR spectra in attenuated total reflection (ATR) mode is obtained with $2 \mathrm{~mL}$ of the sample solution using a ZnSe ATR cell in an FTIR instrument, meanwhile the nanomechanical IR spectra is obtained with $50 \mathrm{pL}$ of the sample solutions (results are available in the ESI $\dagger$ ). Additionally, the nanomechanical IR spectra of four other liquid and solid phase samples (isopropanol, $n$-hexadecane, naphtha, and paraffin wax) are provided in the $\mathrm{ESI} \dagger$ in order to further demonstrate the capability of the technique.

To illustrate the capability of the technique for use in combined chemical and physical analysis, we measured the nanomechanical IR spectra of ethanol-water binary mixture solutions using different concentrations of ethanol. Starting with a $5 \mathrm{wt} \%$ ethanol solution, the BMC is irradiated with IR light from $1180 \mathrm{~cm}^{-1}$ to $1000 \mathrm{~cm}^{-1}$ using MIRCat ${ }^{\mathrm{TM}}$. All ethanol-water binary solutions exhibit strong peaks at $1083 \mathrm{~cm}^{-1}$ and $1045 \mathrm{~cm}^{-1}$ revealing $\mathrm{C}-\mathrm{O}-\mathrm{H}$ bending and $\mathrm{C}-\mathrm{O}$ stretching, respectively (Fig. 3a). By keeping all experimental conditions identical, our results show that the amplitude of the BMC deflection is directly proportional to the sample concentration. As in the ampicillin measurements, there is a linear relationship between the peak amplitude and the concentration of ethanol (inset Fig. 3a). This trend can be extrapolated in order to determine the concentration of ethanol in an ethanol-water mixture solution. Fig. $3 \mathrm{~b}$ shows the relationship between the density of the binary mixture solutions and the resonance frequency of the BMC. More details on density measurements are explained in the ESI. $\dagger$

Previously, a microchannel cantilever was used to measure the density and viscosity of some specific oil samples. ${ }^{13}$ In petroleum exploration industries, the orthogonality of the BMC (proven by ethanol-water experiments) can be exploited to precisely monitor the quality of oil by simultaneously measuring its density, viscosity and chemical composition (proportion of hydrocarbons).

\section{Conclusion}

Our technique of determining the molecular signature of a reagent employed standard technologies. Following a modular 

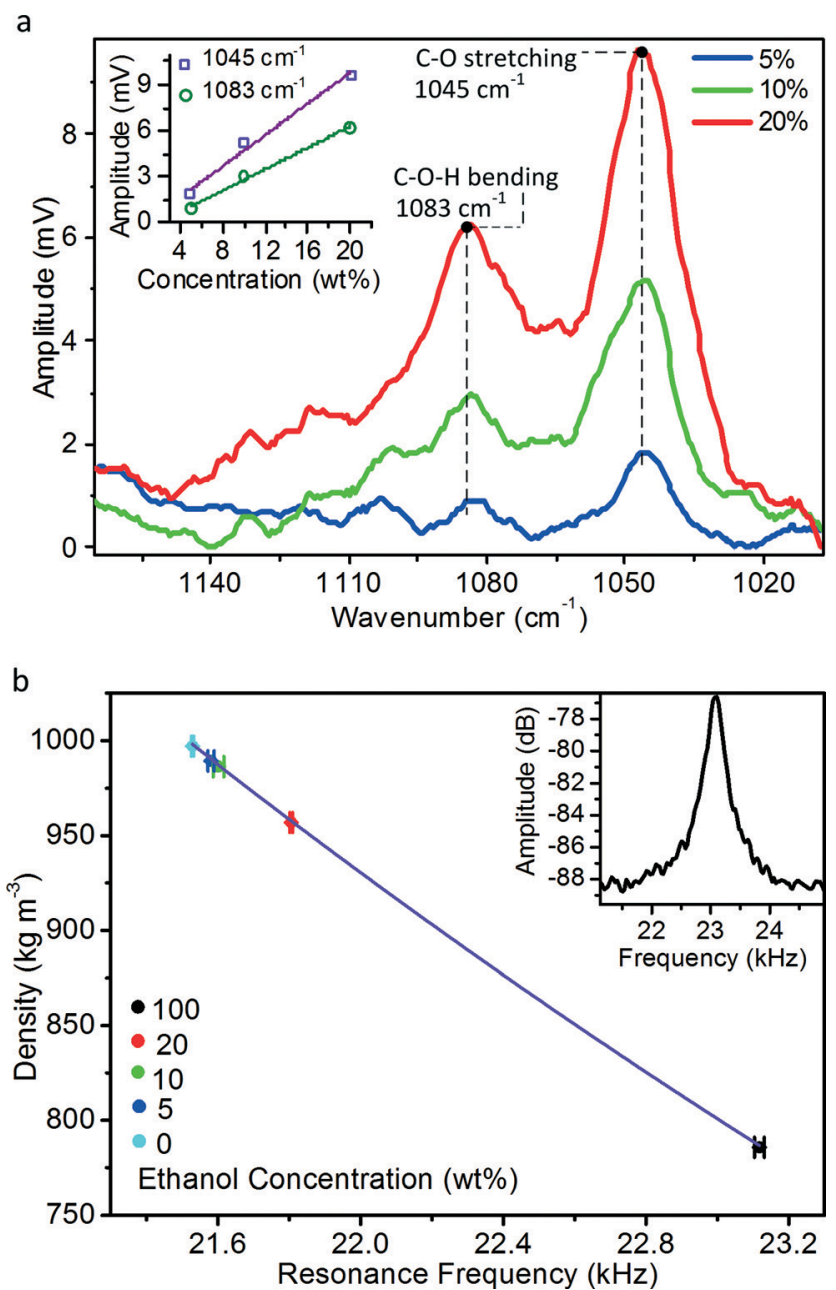

Fig. 3 (a) The sensitivity of the BMC is quantitatively tested by measuring the nanomechanical IR spectra of 50 picoliters of different concentrations (wt\%) of ethanol in ethanol-water binary mixture solutions. By keeping the experimental conditions constant, a strong dependence of IR absorption (BMC deflection amplitude) on the concentration of the analyte is observed. The inset shows a linear relationship between the concentration of ethanol and the deflection amplitude of the BMC. (b) Changes in the resonance frequency are measured with respect to the density of the binary mixture solutions. The solid line shows a fit of eqn. (S2) in the ESI to the data. The inset shows a Fourier spectrum of the fundamental flexural vibration mode of the BMC with $100 \%$ ethanol at $23.1 \mathrm{kHz}$.

approach, the BMC can become a part of a microfluidic motherboard. ${ }^{27,28}$ The nanomechanical detection of molecular signatures of small volumes of confined liquid samples offers interesting possibilities in adding selectivity in $\mu$-TAS and microfluidic devices. Regardless of the light source (ultraviolet, visible or IR), the BMC can be effectively used as a miniature spectrometer to obtain optical absorption spectra of picoliter volumes from liquid samples or mixtures. From the resonance frequency and the quality factor of the BMC, the mass density and viscosity of the liquid can also be obtained simultaneously, making the device a multi-modal system capable of providing orthogonal physical as well as chemical spectroscopic information. As a result of their ability to be mass produced and their miniature size, BMC chips can be used in an array configuration to assess multiple orthogonal measurements (nanomechanical IR spectrum, mass density, viscosity) of numerous analytes at one time.

Significant and intriguing applications, such as drug discovery, ${ }^{29}$ direct monitoring of products from micro-bioreactors ${ }^{22}$ and the study of chemical composition of microbes/cells ${ }^{30}$ are anticipated by the future integration of more sophisticated microfluidics into our system.

\section{Acknowledgements}

This work was supported by the V. Kann Rasmussen (VKR) Foundation (Denmark) and the Canada Excellence Research Chairs (CERC) Program.

\section{Notes and references}

1 A. Arora, G. Simone, G. B. Salieb-Beugelaar, J. T. Kim and A. Manz, Anal. Chem., 2010, 82, 4830-4847.

2 L. Mazutis, J. Gilbert, W. L. Ung, D. A. Weitz, A. D. Griffiths and J. A. Heyman, Nat. Protoc., 2013, 8, 870.

3 A. W. Browne, L. Ramasamy, T. P. Cripe and C. H. Ahn, Lab Chip, 2011, 11, 2440-2446.

4 C. H. Chen, Y. Lu, M. L. Y. Sin, K. E. Mach, D. D. Zhang, V. Gau, J. C. Liao, C. Joseph and P. K Wong, Anal. Chem., 2010, 82, 1012-1019.

5 P Enoksson, G. Stemme and E. Stemme, Sens. Actuators, A, 1995, 47, 327.

6 T. P. Burg and S. R. Manalis, Appl. Phys. Lett., 2003, 83, 2698-2700.

7 T. P. Burg, M. Godin, S. M. Knudsen, W. Shen, G. Carlson, J. S. Foster, K. Babcock and S. R. Manalis, Nature, 2007, 446, 1066-1069.

8 S. Son, W. H. Grover, T. Burg and S. R. Manalis, Anal. Chem., 2008, 80, 4757-4760.

9 M. Godin, F. F. Delgado, S. Son, W. H. Grover, A. K. Bryan, A. Tzur, P. Jorgensen, K. Payer, A. D. Grossman, M. W. Kirschner and S. R. Manalis, Nat. Methods, 2010, 7, 387-390.

10 S. Son, A. Tzur, Y. Weng, P. Jorgensen, J. Kim, M. W. Kirschner and S. R. Manalis, Nat. Methods, 2012, 9, 910-912.

11 P. Enoksson, G. Stemme and E. Stemme, Sens. Actuators, A, 1996, 54, 558-562.

12 I. Lee, K. Park and J. Lee, Sens. Actuators, A, 2013, 194, 62-66.

13 M. F. Khan, S. Schmid, P. E. Larsen, Z. J. Davis, W. Yan, E. H. Stenby and A. Boisen, Sens. Actuators, B, 2013, 185, 456-461.

14 T. P. Burg, J. E. Sader and S. R. Manalis, Phys. Rev. Lett., 2009, 102, 228103.

15 I. Lee, K. Park and J. Lee, Rev. Sci. Instrum., 2012, 83, 116106.

16 S. Kim, D. Lee, X. Liu, C. Van Neste, S. Jeon and T. Thundat, Sci. Rep., 2013, 3, 1111.

17 X. Yao, X. Lib, F. Toledob, C. Zurita-Lopezb, M. Gutovab, J. Momandb and F. Zhoub, Anal. Biochem., 2006, 2, 220-228. 
18 G. Zheng, X. P. A. Gao and C. M. Lieber, Nano Lett., 2010, 8, 3179-3183.

19 M. G. von Muhlen, N. D. Brault, S. M. Knudsen, S. Jiang and S. R. Manalis, Anal. Chem., 2010, 5, 1905-1910.

20 Y. Weizmann, F. Patolsky and I. Willner, Analyst, 2001, 126, 1502-1504.

21 H. Zhang, E. C. Yi, X. Li, P. Mallick, K. S. Kelly-Spratt, C. D. Masselon, D. G. Camp, R. D. Smith, C. J. Kemp and R. Aebersold, Mol. Cell. Proteomics, 2005, 4, 144-155.

22 N. Szita, P. Boccazzi, Z. Zhang, P. Boyle, A. J. Sinskey and K. F. Jensen, Lab Chip, 2005, 5, 819-826.

23 T. M. Mata, A. A. Martinsa and N. S. Caetanob, Renewable Sustainable Energy Rev., 2010, 14, 217-232.

24 M. Iero, R. Valenti, V. Huber, P. Filipazzi, G. Parmiani, S. Fais and L. Rivoltini, Cell Death Differ., 2008, 15, 80-88.
25 S. J. Shire, Z. Shahrokh and J. Liu, J. Pharm. Sci., 2004, 6, 1390-1402.

26 M. F. Khan, S. Schmid, Z. J. Davis and A. Boisen, Microelectron. Eng., 2011, 8, 2300-2303.

27 J. W. Choi, K. W. Oha, J. H. Thomasb, W. R. Heinemanb, H. B. Halsallb, J. H. Nevina, A. J. Helmickia, H. T. Hendersona and C. H. Ahn, Lab Chip, 2002, 2, 27-30.

28 A. Heiskanen, V. Coman, N. Kostesha, D. Sabourin, N. Haslett, K. Baronian, L. Gorton, M. Dufva and J. Emnéus, Anal. Bioanal. Chem., 2013, 405, 3847-3858.

29 P. S. Dittrich and A. Manz, Nat. Rev. Drug Discovery, 2006, 5, 210-218.

30 F. F. Delgado, N. Cermak, V. C. Hecht, S. Son, Y. Li, S. M. Knudsen, S. Olcum, J. M. Higgins, J. Chen, W. H. Grover and S. R. Manalis, PLoS One, 2013, 8, e67590. 